\title{
ANALYSIS OF DYNAMICS AND FRICTION RESISTANCE IN THE CAM-TAPPET SYSTEM
}

\author{
KRZYSZTOF SICZEK \\ Lodz University of Technology, Department of Vehicles and Fundamentals of Machine Design, Eódź, Poland \\ e-mail: krzysztof.siczek@p.lodz.pl \\ ANDRZEJ STEFAŃsKi \\ Lodz University of Technology, Division of Dynamics, Eódź, Poland \\ e-mail: andrzej.stefanski@p.lodz.pl
}

\begin{abstract}
In this paper, the influence of friction resistance and mutual contact interaction on dynamical properties of the cam-tappet mechanism is analyzed. A dynamical model of the cam-tappet contact has been developed. Chosen results of numerical simulations of this model are presented for cases with and without oil lubrication in contact zones. Various phenomena accompanying the cam-tappet contact dynamics have been observed, e.g., change of the global direction of tappet rotation, local oscillation of these revolutions, changes of friction in function of frequency of the camshaft longitudinal vibrations. We confirm, that the growing amplitude of camshaft longitudinal vibration causes an increase in the tappet rotational speed, whereas its reducing to small values leads to stopping the tappet rotation.
\end{abstract}

Keywords: cam-tappet mechanism, friction, lubrication

\section{Introduction}

Many machines like combustion engines employ mechanisms forced by a roller or plane tappet driven by cam devices. A characteristic feature observed in such mechanisms is the occurrence of concentrated line contact between the touching surfaces. There exist conditions of elastohydrodynamic lubrication (EHL) allowing variations of the friction type from the mixed to the boundary one. The contact zone is loaded by the force changing both, in value and its acting direction. The main component of the sliding velocity which results from the mechanism operational cycle also varies. During the time when both surfaces remain in contact, additional relative displacements, small in amplitude, may occur in peripheral, axial and normal directions of the cam motion. They may be a result of torsional vibrations of the camshaft. The axial displacements may result from bending vibrations and from forced displacement within the axial clearance. Such displacements being normal to the contacting surfaces may arise from changes of the loading force and bending vibrations of the camshaft. Obviously, they change the course of contact loading.

The amount of friction between two lubricated sliding surfaces depends primarily on the contacting materials, load, lubricant formulations and the lubrication regimes. Under boundary and mixed lubrication conditions, where some asperities of both surfaces touch each other, friction can be controlled by lubricant formulations and appropriate surface engineering.

Most studies on cam-follower contacts are addressed to friction and wear measurements for investigating the influence of lubricant additives and surface coatings, finish and texture, see Willermet et al. (1991), Soejima et al. (1999), Lindhom and Svahn (2006), Kano (2006), Lewis and Dwyer-Joyce (2002). The friction force (or torque) is often calculated by subtracting the 
contributions of other components and inertia actions from the measured values, as in Kano (2006), Baş et al. (2003).

The action of friction forces between the cam and tappet or the valve stem can cause tappet or valve rotations. Their effects can become positive, as they decrease friction resistance between contacting surfaces. The rotations are forced when the symmetry plane of the cam is displaced relative to the tappet or valve axis. Such behavior was described in Jelenschi et al. (2011). For a particular application, a specific amount of valve rotation is required to maintain even the sealing level at the valve seat. Excessive amount of valve rotation will result in increased wear of the contacting surfaces, limiting the engine lifetime. In the analysis described in Refalo et al. (2010), a minimum engine speed of $3500 \mathrm{rpm}$ was established as a target for valve rotation to begin. Over a typical driving cycle, the valves can rotate at least once. The maximum value of valve rotation can reach $15 \mathrm{rpm}$ for any engine speed. Typical factors influencing valve rotation include oil temperature, assembly variation, machining variability, valve deposits, engine vibrations and engine temperature.

Hyundai in Kim et al. (2004) are described what influenced and contributed to valve rotation. It was explained that during opening the valve also rotated along with the valve spring. Next, during the closing phase, the valve moved back to its original position. As the speed of the motion increased, there was a greater tendency for the valve to slide at both - the maximum lift and the closing events. This sliding motion provides a rotational net movement over a given lift event. All this motion is balanced by friction between each of the contact interfaces: between the cylinder head, valve spring, valve, retainer, lock, drive mechanism, etc.

In the research described in Hiruma and Furuhama (1978) it was observed that such a valve started to rotate after reaching the crankshaft speed of $3000 \mathrm{rpm}$. While speeding up the crankshaft, the valve rotation increased rapidly. Before reaching the level of $3000 \mathrm{rpm}$ at the crankshaft, the valve did not rotate or it rotated in varying directions. Also in Beddoes (1992) similar behavior of random nature and different directions of valve rotation was reported.

The main aim of this paper is to check if the occurrence of camshaft longitudinal vibrations can decrease friction between the cam and tappet of the valve tip. Also analysis of dynamics of cam-tappet contact is discussed.

The paper is organized as follows. In Section 2, profiles of the cam mechanism are briefly described and illustrated. In Section 3, the dynamical model of the cam-tappet contact is introduced. Results of numerical simulations of the cam-tappet dynamics in the case with and without oil lubrication in the contact zones are demonstrated in Section 4. Finally, Section 5 contains discussion of the obtained results and conclusions.

\section{Valve lift profiles in the cam mechanism}

A very aggressive cam profile is the best when used with bucket tappets, as stated in Blair et al. (2005). The base circle radius $R_{b}$ of the cam equals $0.02 \mathrm{~m}$ and the valve (and bucket tappet by definition) stroke is $8.3 \mathrm{~mm}$. The bucket tappet is assumed flat. The bucket has the minimum possible diameter $(d t=0.03 \mathrm{~m})$ in order to keep the declared width of the cam in full contact conditions with the flat tappet surface throughout the working cycle. The design of cam profiles was presented in Rothbart (2004). They can have a polynomial form

$$
\begin{aligned}
h= & h_{\max }\left[1+C_{2}\left(\frac{\theta_{c}-\theta_{c}\left(h_{\max }\right)}{\beta}\right)^{2}+C_{p}\left(\frac{\theta_{c}-\theta_{c}\left(h_{\max }\right)}{\beta}\right)^{p}+C_{q}\left(\frac{\theta_{c}-\theta_{c}\left(h_{\max }\right)}{\beta}\right)^{q}\right. \\
& +C_{r}\left(\frac{\theta_{c}-\theta_{c}\left(h_{\max }\right)}{\beta}\right)^{r}+C_{s}\left(\frac{\theta_{c}-\theta_{c}\left(h_{\max }\right)}{\beta}\right)^{s}
\end{aligned}
$$

where $p=22, q=42, r=62, s=82$ is a constant power and $C_{2}=-p q r s[(p-2)(q-2)(r-$ $2)(s-2)]^{-1}, C_{p}=2 q r s[(p-2)(q-2)(r-p)(s-p)]^{-1}, C_{q}=-2 p r s[(q-2)(q-p)(r-q)(s-q)]^{-1}$, 
$C_{r}=2 p q s[(r-2)(r-p)(r-q)(s-r)]^{-1}, C_{s}=-2 p q r[(s-2)(s-p)(s-q)(s-r)]^{-1}$ are constant factors, $\beta=\pi / 3$ - angle of rise, $h_{\max }$ - cam stroke.
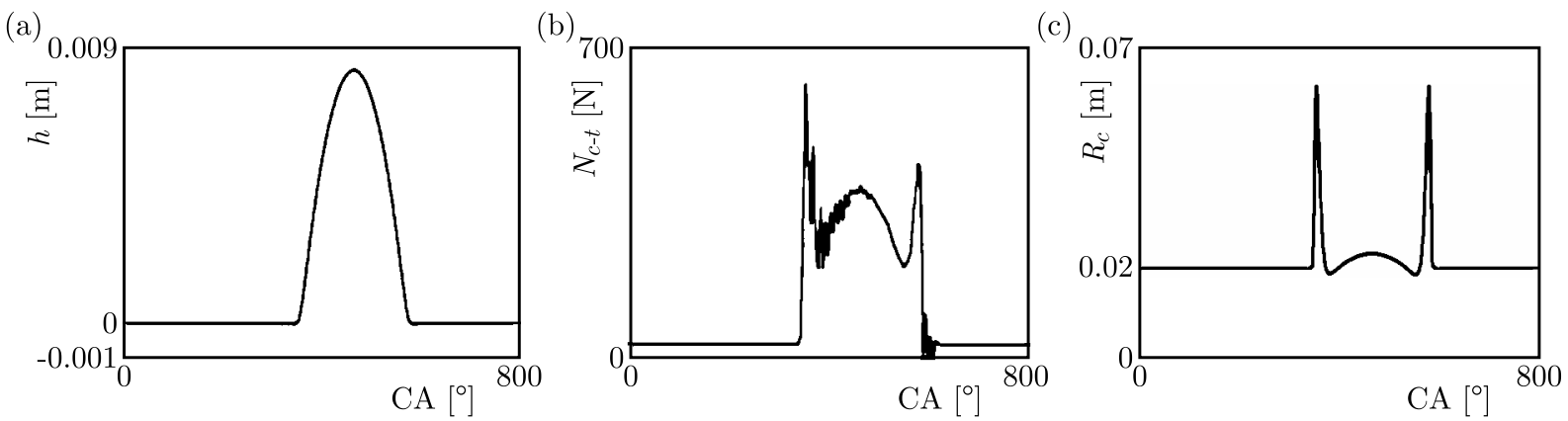

Fig. 1. (a) Diagram of thevalve lift $h$ against the crankshaft angle $\theta_{c}=C A$ (crankshaft angle degree).

(b) Diagram of the normal force $N_{c-t}$ between the cam and tappet against the crankshaft angle $\theta_{c}=C A$. (c) The instantaneous radius of curvature $R_{c}$ of the cam profile against the crankshaft angle $\theta_{c}=C A$

The shape of the valve lift $h$ against the crankshaft angle $\phi=C A$ is presented in Fig. 1a. The typical trace of the normal force $N_{c-t}$ between the cam and tappet against $\phi=C A$ has been assumed to be similar as that in Taraza et al. (1999) for the crankshaft speed $1320 \mathrm{rpm}$, and is presented in Fig. 1b. The instantaneous radius of curvature $R_{c}$ of the cam profile, shown in Fig. 1c, can be calculated from the formula

$$
R_{c}=R_{b}+h+\frac{a}{\omega_{c}^{2}}
$$

where $R_{b}$ is the base radius of cam, $\omega_{c}$ - angular velocity of the crankshaft, $a=d^{2} l / d t^{2}, l(t)-$ varying displacement (Fig. 2b).

\section{Modelling of the cam-tappet contact}

Mathematical models allowing prediction of lubricant film-thickness and Hertzian pressures at the cam/tappet contact were presented in Gecim (1992). The model can predict the changes in the cam/tappet interface friction due to changing operating conditions. Also a model of tappet spin allowing for slip at the cam/tappet interface has been included. Modelling the tappet spin allows one to see the effects of the tappet crown radius and cam-taper angle on the interface frictional loss. It is found that tappet rotation is affected by design and operating conditions, and depends primarily on camshaft speed.

The use of advanced mathematical models to quantify power loss at cam/tappet contact, tappet/bore contact and camshaft bearings was presented in Calabretta et al. (2010). Calculated and measured friction data for the valve train of a high speed passenger car engine were compared with those obtained from tests on a motored cylinder head test rig. The system friction was measured and calculated across the operating speed range with different oil supply temperature.

The camshaft model considering both camshaft angular vibration and bending vibration was presented in Guo et al. (2015). Each follower element was treated as a multi-mass system. The lumped masses were connected by spring elements and damping elements. The contact force model at the cam-tappet interfaces was developed based on the elasto-hydrodynamic lubrication theory of finite line conjunction. It was that bending vibration of the camshaft was mainly in the normal direction at the cam-tappet interfaces. Bending vibration was mainly influenced by overlapping of the inlet and exhaust cam functions of each cylinder. The angular vibration of the camshaft mainly focused at the fundamental frequency and the harmonic frequency corresponding to the cylinder number. 


\subsection{Description of the model}

The present dynamic model of the tappet-cam assembly is shown in Fig. 2a for the camshaft oscillating in the $z$ direction and rotating about such an axis with a constant angular velocity $\omega_{c}$. The tappet can rotate about its axis with the angle $\varepsilon$. It is assumed that friction torque $M_{T t-p}$ between the bucket tappet and the valve stem of diameter $d$ or between the tappet and pushrod of cross-section diameter $d_{p}$ can be estimated as follows

$$
M_{T t-p} \approx \frac{1}{3} \mu_{t-p} N_{c-t} d_{p}
$$

where $\mu_{t-p}$ is the friction coefficient between the tappet and pushrod, which may vary depending on the amount of oil, sliding velocity of the pushrod against the tappet, and $N_{c-t}$ represents the load of contact zone equal to the normal force between the cam and tappet (Fig. 2b). The friction between the tappet and pushrod is complex and of a mixed type, and sometimes even of the boundary type, as wear debris and pollution can cumulate in the contact zone. The friction coefficient can also vary with the loading in the contact zone and vibration amplitude. In order to ignore the effect of variation of the friction coefficient $\mu_{t-p}$ on motion of the tappet, only a constant value of that coefficient has been considered. For simplicity of calculations, it has been assumed a constant value of the friction coefficient $\mu_{t-p}$ equal to the averaged one 0.2 , and diameter $d_{p}=0.006 \mathrm{~m}$. The friction torque $M_{T c-t}$ between the cam and tappet is calculated from the equation

$$
M_{T c-t}=\mu_{c-t} N_{c-t} A_{c 1} \sin \left(2 \pi f_{c 1} t\right)
$$
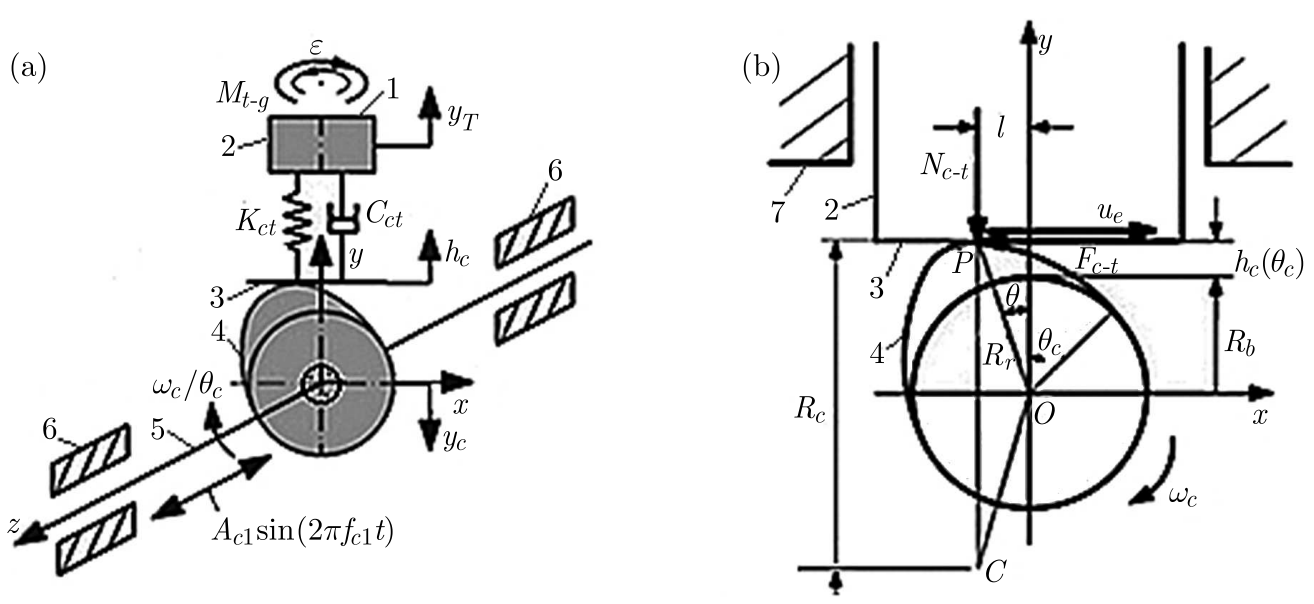

Fig. 2. (a) Dynamic model of the tappet-cam assembly for the camshaft oscillating in the $z$ direction and rotating about such an axis. (b) The scheme of the cam-tappet contact; 1 - contact surface between the tappet and pushrod, 2 - contact surface between the tappet and its guide, 3 - plane parallel to the tappet frontal face and tangent to the cam surface, 4 - cam surface, 5 - camshaft, 6 - camshaft bearing,

$$
7 \text { - tappet guide }
$$

Longitudinal oscillations of the camshaft along the $z$ axis have the amplitude $A_{c 1}$ and frequency $f_{c 1}$. The friction coefficient $\mu_{c-t}$ between the cam and tappet is complex and varies as described further. The friction coefficient $\mu_{t-g}$ between the tappet and guide varies depending on the amount of oil, sliding velocity of the tappet against its guide and load ib the contact zone. For simplicity of calculations, it has been assumed a constant value of friction coefficient $\mu_{t-g}$ equal to the averaged one, being 0.15 . The friction torque $M_{T t-g}$ between the tappet and its guide is calculated from

$$
M_{T-g}=\frac{1}{2} \mu_{t-g} F_{c-t} d_{t}=\frac{1}{2} \mu_{t-g} \mu_{c-t} N_{c-t} d_{t}
$$


where diameter of the tappet equals $d_{t}=0.03 \mathrm{~m}$. The balance of torques acting on the tappet is described by a dynamical differential

$$
I_{y} \ddot{\varepsilon}+M_{T t-g}+M_{T t-p}=M_{T c-t}
$$

where $I_{y}$ is the mass moment of inertia of the tappet with respect to the axis $Y$ (see Fig. $2 \mathrm{~b}$ ). Substituting Eqs. (3.1)-(3.3) into Eq. (3.4), we have

$$
I_{y} \ddot{\varepsilon}+\frac{1}{2} \mu_{t-g} \mu_{c-t} N_{c-t} d_{t}+\frac{1}{3} \mu_{t-p} N_{c-t} d_{p}=\mu_{c-t} N_{c-t} A_{c 1} \sin \left(\omega_{c 1} t\right)
$$

where $\omega_{c 1}=2 \pi f_{c 1} t$.

The initial conditions correspond to the situation of the rest, i.e., $\varepsilon(t=0)=0, \dot{\varepsilon}(t=0)=0$.

\subsection{Lubrication in the contact zone between the cam and tappet}

When the camshaft rotates and undergoes axial oscillations, the oil flow in the contact zone between the cam and tappet becomes very complex. The cam motion against the tappet caused only by camshaft rotation allows the occurrence of an oil film over the contact zone between the tappet and cam. This film is characterized by varying thickness and dominant fluid motion in the periphera $\theta$ direction. The characteristic central thickness of such an oil film is equal to $h_{0}$. Estimation of thickness $h_{0}$ is described in Section 3.3. The cam motion against the tappet caused only by camshaft longitudinal oscillations also results in varying thickness of the oil film in the mentioned contact zone. However, the dominant fluid motion in the contact zone is in the axial $z$ direction. Averaged oil thickness of this oil film is equal to $h_{v}$. Estimation of thickness $h_{v}$ is described in Section 3.4. If camshaft motions occur both due to rotation and longitudinal oscillations, the resulted oil film thickness $h$ is higher than both $h_{0}$ and $h_{v}$. In real, the existence of such two camshaft motions influences the oil thickness $h$ in a very complex manner but, for simplicity, it can be treated as a superposition. Therefore, it has been assumed that the oil film thickness $h \approx h_{0}+h_{v}$, where $h_{0}$ is the central oil film thickness due to camshaft rotation, $h_{v}$ - averaged oil film thickness due to camshaft longitudinal oscillation. When both rotation and axis oscillation of the camshaft occurs, the resulted normal force $N_{c-t}^{r e s}$ can be estimated from the equation given in Section 3.3 and corresponding to the force $N_{t-c}$. Also the resulted friction force $F_{c-t}^{r e s}$ in this zone can be obtained from the equations given in Section 3.3 and corresponding to the force $F_{c-t}$. In both these cases, the thickness $h_{0}$ is substituted by $h$. Then the friction coefficient $\mu_{c-t}^{r e s} \mu_{c-t}$ between the tappet and pushrod is estimated from the equation

$$
\mu_{c-t}^{r e s}=\frac{N_{c-t}^{r e s}}{T_{c-t}^{r e s}}
$$

\subsection{Elasto-hydrodynamic lubrication and the contact force model due to rotation}

High pressure developed between the cam profile and the tappet requires conditions of elasto-hydrodynamic (EHD) lubrication in the contact zone. At high pressure, the viscosity of the oil increases exponentially with pressure, and an oil film can be maintained between the cam and the tappet, as described in Teodorescu et al. (2003). The minimum oil thickness exists at the oil exit, and the oil film can be assumed to stay nearly parallel along the lubricated zone. So the oil film thickness can be estimated at the centre point of the lubricated zone. Calculation of the central oil film thickness can be carried out from the equation

$$
h_{0}^{*}=1.67 G^{* 0.421} U^{* 0.541} W^{* 0.059} \exp \left(-96.775 w_{s}^{*}\right)
$$


developed by Rahnejat, and described in Teodorescu et al. (2005, 2007), Kushwaha et al. (2000), for finite line concentrated contact conjunction for combined entraining and squeeze film actions. The dimensionless parameters in Eq. (3.7) are described by the following formulas

$$
\begin{array}{ll}
h_{0}^{*}=\frac{h_{0}}{R_{C}} \quad G^{*}=\alpha_{1} E_{C} & U^{*}=u_{e} \eta_{0}\left[E_{C} R_{C}\right]^{-1} \\
W^{*}=N_{c-t}\left[E_{C} R_{C} L_{C}\right]^{-1} & w_{s}^{*}=\frac{\dot{h}_{0}}{u_{e}}
\end{array}
$$

where $h_{0}$ is the central oil film thickness, $\alpha_{1}$ - pressure viscosity coefficient, $\eta_{0}$ - dynamic viscosity at the inlet of contact, $u_{e}$ - oil entraining velocity, $E_{C}$ - effective elastic modulus, $N_{c-t}$ - normal load responsible for local deformation of the cam/tappet contact, $R_{C}$-instantaneous radius of curvature, $L_{C}=0.014 \mathrm{~m}$ - cam width. The symbol $w_{s}^{*}$ represents the squeeze-roll ratio, and its range of applicability is given, by Rahnejat, between 0 and 0.005 , and in the present analysis it is assumed to be constant and equal to 0.005 . The effective modulus can be calculated from the equation

$$
E_{C}^{-1}=\frac{1}{2}\left[\left(1-\nu_{1}^{2}\right) E_{1}^{-1}+\left(1-\nu_{2}^{2}\right) E_{2}^{-1}\right]
$$

where $E_{1}=210 \mathrm{GPa}$ is the elastic modulus of the cam material, $E_{2}=210 \mathrm{GPa}$ - elastic modulus of the tappet material, $\nu_{1}=0.3$ - Poisson's ratio of the cam material, $\nu_{2}=0.3$ - Poisson's ratio of the tappet material.

The oil entraining velocity $u_{e}$ is calculated according to the formula

$$
u_{e}=\frac{1}{2} \omega_{c}\left(R_{b}+h(\theta)+2 a(\theta)\right)
$$

The value of $N_{c-t}$ can be approximated using the following equation presented in Guo et al. (2011)

$$
N_{c-t}=K_{c-t}\left(h(\theta)-y_{C}-y_{T}\right)+C_{c-t}\left(v(\theta)-\dot{y}_{C}-\dot{y}_{T}\right)
$$

where $y_{C}$ is the displacement of camshaft bending motion, $y_{T}$ - displacement of the tappet, $K_{c-t}=1.434 \cdot 10^{8} \mathrm{~N} / \mathrm{m}, C_{c-t}=115.292 \mathrm{Ns} / \mathrm{m}-$ contact stiffness and damping coefficient between the cam and tappet, respectively, as given in Guo et al. (2011). Values of $y_{C}$ and $y_{T}$ are not known, so their sum can be estimated in the following manner. Let us assume that the course of the cam force $F$ against time $t$ is the same as the course of $N_{c-t}$. Hence, we have the relationship

$$
K_{c-t}\left(y_{C}+y_{T}\right) \gg C_{c-t}\left(\dot{y}_{C}+\dot{y}_{T}\right)
$$

so, from Eq. (3.3), the value of $\left[y_{C}+y_{T}\right](\theta)$ can be estimated. It is represented by the approximate formula

$$
\left[y_{C}+y_{T}\right](\theta) \approx\left[K_{c-t} h(\theta)+C_{c-t} v(\theta)-N_{c-t}(\theta)\right] \frac{1}{K_{c-t}}
$$

Then the sum of velocities $\left[\dot{y}_{C}+\dot{y}_{T}\right](\theta)$ can be estimated from the equation

$$
\left[\dot{y}_{C}+\dot{y}_{T}\right](\theta)=\omega_{C} \frac{\partial\left[y_{C}+y_{T}\right](\theta)}{\partial \theta}
$$

and finally the corrected form of $N_{c-t}$ can be calculated from equation (3.11). The friction force $F_{c-t}$ between the cam and tappet is due to two different mechanisms, the asperity contact (boundary part $T_{b}$ ) and the shear of lubricant (hydrodynamic part $T_{v}$ ), as described in Teodorescu et al. (2003, 2005), Yang et al. (1996). The asperity interaction model is based on the theory 
developed by Greenwood and Tripp (1971). The boundary friction $T_{b}$ was determined by Guo et al. (2011)

$$
T_{b}=\tau_{0} A_{a}+m P_{a}
$$

where $\tau_{0}=2.0 \mathrm{MPa}$ is the Eyring shear stress, described in Rothbart (2004), $m=0.17$ - pressure coefficient of the boundary shear strength, described in Guo et al. (2011). Considering a Gaussian distribution of the asperities heights and fixed asperity radius of curvature, the area $A_{a}$ occupied by the asperity peaks and the load $P_{a}$ carried by the asperities are calculated as in Guo et al. (2011)

$$
A_{a}=\pi^{2}\left(\zeta \beta \sigma_{R}\right)^{2} A F_{2}(\lambda) \quad P_{a}=\frac{8 \sqrt{2}}{15} \pi\left(\zeta \beta \sigma_{R}\right)^{2} \sqrt{\frac{\sigma_{R}}{\beta}} E_{C} A F_{5 / 2}(\lambda)
$$

where $\zeta$ is the asperity density, $\beta$ - radius of curvature, $\sigma_{R}=0.4 \mu \mathrm{m}$ - composite surface roughness parameter, $A$ - Hertzian contact area, $\lambda=h_{0} / \sigma_{R}$ - constant. It has been assumed that $\left(\zeta \beta \sigma_{R}\right)=0.055$ and $\sigma_{R} / \beta=0.001$, as in Guo et al. (2011). The Hertzian formula for the contact of two cylinders can be used to calculate the contact area, see Patir and Cheng (1979)

$$
E_{C} A=\sqrt{\frac{8}{\pi} E_{C} R_{C} L_{C} N_{c-t}}
$$

Two statistical functions $F_{2}(\lambda)$ and $F_{5 / 2}(\lambda)$ are defined by the equation

$$
F_{n}(\lambda)=\frac{1}{2 \pi} \int_{h_{0} / \sigma}^{\infty}\left(s-\frac{h_{0}}{\sigma_{R}}\right)^{n} \exp \left(-\frac{1}{2} s^{2}\right) d s
$$

They can be approximated by the following formulas

$$
\begin{aligned}
& F_{2}(\lambda)=-0.0018 \lambda^{5}+0.0281 \lambda^{4}-0.1728 \lambda^{3}+0.5258 \lambda^{2}-0.8043 \lambda+0.5003 \\
& F_{5 / 2}(\lambda)=-0.0046 \lambda^{5}+0.0574 \lambda^{4}-0.2958 \lambda^{3}+0.7844 \lambda^{2}-0.0776 \lambda+0.6167
\end{aligned}
$$

The viscous friction is given by

$$
T_{v}=\tau\left(A-A_{a}\right)
$$

where $\tau$ is the shear stress of the lubricant. Depending on the oil film thickness, the lubricant may behave as a Newtonian or non-Newtonian oil film, as described in Teodorescu et al. (2003). The behavior can be estimated by the Eyring shear stress $\tau_{0}$. If the shear stress is lower than the Eyring shear stress $\tau_{0}$ then Newtonian behavior occurs, otherwise non-Newtonian behavior takes place. So the shear stress can be expressed by the equation presented in Guo et al. (2011)

$$
\tau=\left\{\begin{array}{lll}
\frac{\eta u_{S}}{h_{0}} & \text { for } & \tau \leqslant \tau_{0} \\
\tau_{0}+\gamma_{S} p^{*} & \text { for } & \tau>\tau_{0}
\end{array}\right.
$$

where $\eta$ is the oil viscosity, $\eta=\eta_{0} \exp \left(\alpha_{1} p^{*}\right), \eta_{0}=0.0057 \mathrm{~Pa} / \mathrm{s}, \alpha_{1}=1.8 \cdot 10^{-8} \mathrm{~m}^{2} / \mathrm{N}, u_{S}-$ sliding velocity, $\gamma_{S}=0.08$ - rate of change of shear stress with pressure, and $p^{*}$ - pressure on the oil film described by the (Moraru, 2005)

$$
p^{*}=\frac{N_{c-t}-P_{a}}{A-A_{a}}
$$

The sliding velocity $u_{S}$ between the cam and tappet is calculated from the equation (Guo et al., 2011)

$$
u_{S}=\omega_{c}\left(R_{b}+h(\theta)\right)
$$

The total friction force $F_{c-t}$ is given by the sum (Guo et al., (2011)

$$
F_{c-t}=T_{b}+T_{v}
$$




\subsection{Elasto-hydrodynamic lubrication and the contact force model due to axis oscillations}

The contact between the tappet and cam is considered as the case of two parallel plates sliding relative to each other. The loading force $N_{c-t}$ is balanced by the load $P_{a 1}$ carried by the asperities and by the hydrodynamic force $P_{v}$ as well as by the squeeze force $P_{S}$ described as

$$
N_{c-t}=P_{a 1}+P_{v}+P_{s}
$$

The load $P_{a 1}$ carried by the asperities can be estimated from the following equation

$$
P_{a 1}=A\left(\frac{h_{v} 0-h_{v}}{c}\right)^{1 / m}
$$

similarly to Yang et al. (1996). It should be remembered that $A=2 b L_{C}$, where $b$ is the Hertz half-width of contact between the cam and tappet. The hydrodynamic force $P_{v}$, the squeeze force $P_{S}$ and the velocity $w$ are defined by formulas presented in Siczek (2016)

$$
P_{v}=\frac{6 \eta A L_{c} K_{p} \psi v}{h_{v}^{2}} \quad P_{s}=\frac{\eta b^{2} A w}{h_{v} 3}
$$

and

$$
w=\dot{h}_{v}=-\left[N_{c-t}-A\left(\frac{h_{v 0}-h_{v}}{c}\right)^{1 / m}-\frac{6 \eta A L_{c} K_{p} \psi v}{h_{v}^{2}}\right] \frac{h_{v}^{3}}{\eta A b^{2}}
$$

where $K_{p}=0.0265, \psi=0.06$ are coefficients characterizing hydrodynamic impacts in the contact zone and $h_{v 0}$ is the oil film thickness, as described in Siczek (2016). The initial value of velocity $v=0$ and the film thickness $h_{v}$ is equal to $h_{v 0}$. The initial film thickness $h_{v 0}$ can be estimated assuming that, in the initial conditions, the load $P_{a 1}$ carried by the asperities is equal to $P_{a}$, see $(3.16)_{2}$. After obtaining the oil film thickness $h_{v}$, the force $N_{c-t}$ results from equations (3.25)-(3.27) and the friction force $T_{c-t}$ in the axial $z$ direction from (3.15)-(3.24) by substituting force $F_{c-t}$ by $T_{c-t}$, thickness $h_{0}$ by $h_{v}$ and velocity $u_{s}$ by $v_{2}$ given by the equation

$$
v_{2}=2 A_{c 1} \pi f \cos \left(2 \pi f_{c 1} t\right)
$$

Then the friction coefficient $\mu_{c-t}$ between the tappet and pushrod is estimated from the equation

$$
\mu_{c-t}=\frac{F_{c-t}}{N_{c-t}}
$$

\section{Results}

The first set of numerically simulated results have been obtained for the case of a constant (dry or mixed) friction coefficient $\mu_{c-t}$ occurring in contact between the cam and tappet, and $\mu_{t-p}$ between the tappet and the pushrod, which are equal to 0.3. In Fig. 3, an exemplary time-diagram of the $F_{c-t}$ force obtained for the camshaft rotational speed $n_{s}=1320 \mathrm{rpm}$, the camshaft longitudinal oscillations of the amplitude $A_{c l}=0.0001 \mathrm{~m}$ and the frequency $f_{c l}=1 \mathrm{~Hz}$, is demonstrated. It is a single segment of the force sequence for a time period of $0.1 \mathrm{~s}$. The entire sequence includes a time period of $60 \mathrm{~s}$. Subsequent time-diagrams of the tappet rotation angle $\varepsilon$ for various values of the frequency $f_{c l}$ are shown in Figs. 4a-d. The case of tappet rotation, corresponding to Fig. 3, is shown in Fig. 4a. An increase in such an angle with time in almost step-like manner can bee observed.

If the frequency $f_{c l}$ is significantly elevated, up to $10 \mathrm{~Hz}$, we can observe almost a linear increase in the angle $\varepsilon$ (see Fig. 4b). Cyclic variations of the direction of tappet rotation occur, 


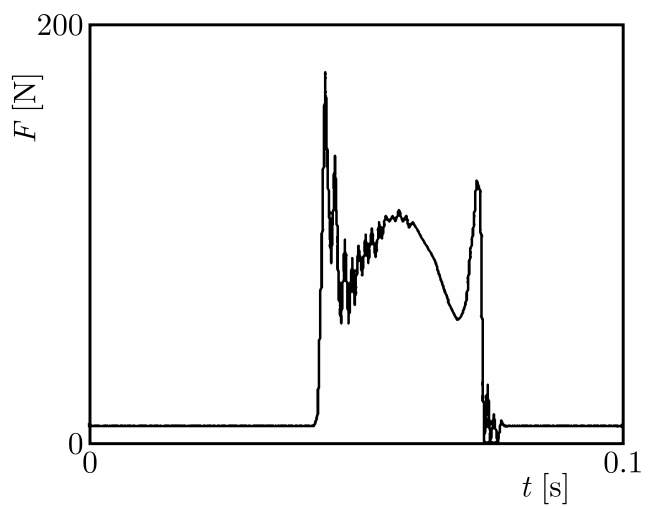

Fig. 3. Time-diagrams of the force $F_{c-t}$ between the cam and the tappet for the frequency of camshaft longitudinal oscillations $f_{c l}=1 \mathrm{~Hz}$. Values of remaining parameters: $\mu_{c-t}=\mu_{t-p}=0.3, n_{s}=1320 \mathrm{rpm}$,

$$
A_{c l}=0.0001 \mathrm{~m}
$$

(a)

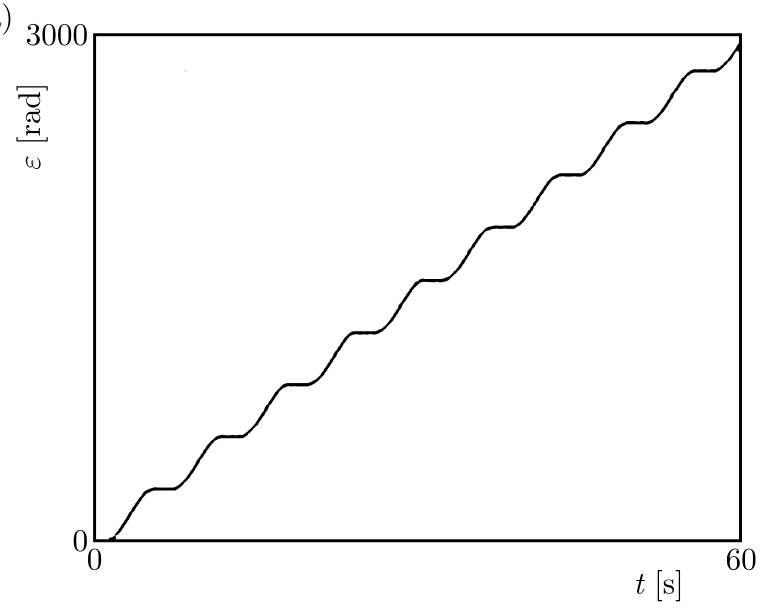

(c)

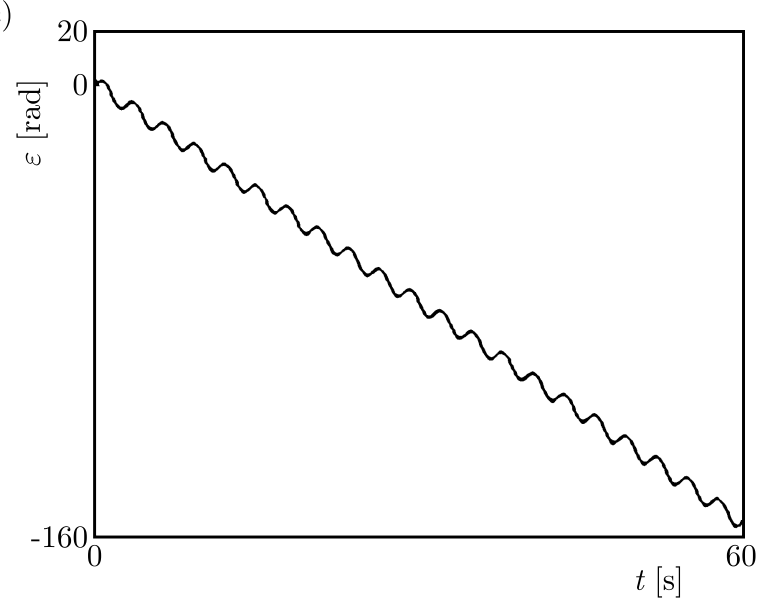

(b)

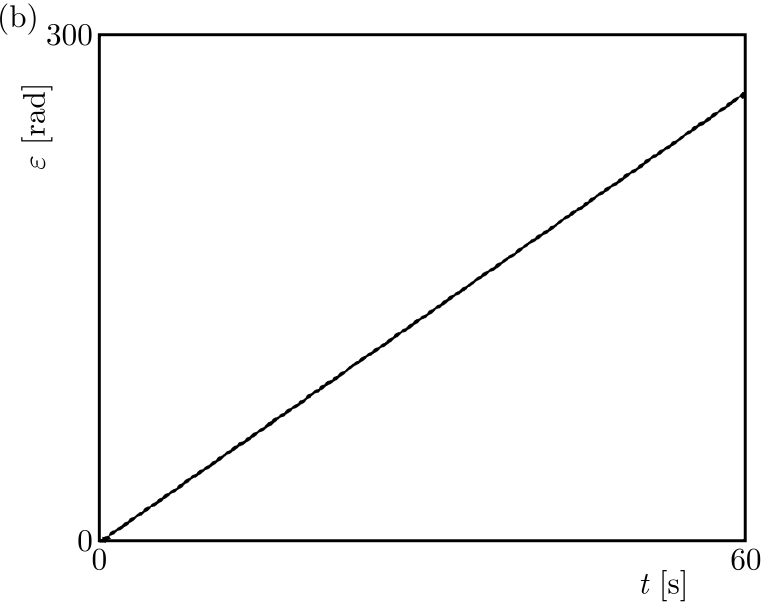

(d)

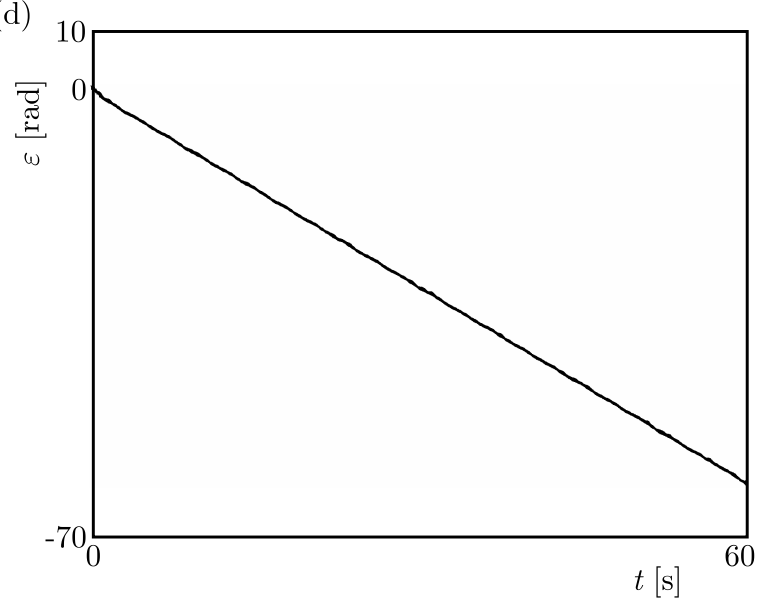

Fig. 4. Time-diagrams of the tappet rotation angle $\varepsilon$ for various frequencies of the camshaft longitudinal oscillations $f_{c l}: 1 \mathrm{~Hz}(\mathrm{a}), 10 \mathrm{~Hz}(\mathrm{~b}), 20 \mathrm{~Hz}$ (c), $100 \mathrm{~Hz}$ (d). Values of remaining parameters: $\mu_{c-t}=\mu_{t-p}=0.3, n_{s}=1320 \mathrm{rpm}, A_{c l}=0.0001 \mathrm{~m}$

but the observed oscillations have a very small amplitude. Further growth of the camshaft vibration frequency $\left(f_{c l}=20 \mathrm{~Hz}\right)$ causes reversal of the direction of tappet rotation. However, as shown in Fig. 4c, cyclic variations of this direction have a quite large amplitude. The same direction of tappet rotation and its increase with time $t$ has been obtained for the frequency $f_{c l}=100 \mathrm{~Hz}$, as it is depicted in Fig. 4d. However, in this case, variations of the tappet rotation direction are very small. 
In the next two diagrams (Figs. 5a and 5b) the influence of the amplitude change on tappet rotation is demonstrated. In the case when the amplitude is doubled $\left(A_{c l}=0.0002 \mathrm{~m}\right)$ and the frequency $f_{c l}=10 \mathrm{~Hz}$, the obtained time history of the tappet rotation angle $\varepsilon$ is shown in Fig. 5a. It can be seen that an increase in the angle $\varepsilon$ with time occurs in the same direction as in the case of $A_{c l}$ equal to 0.0001 , but 4.44 times greater than the last one. On the other hand, reducing the amplitude to a half of the initial value $\left(A_{c l}=0.00005 \mathrm{~m}\right.$ and $\left.f_{c l}=10 \mathrm{~Hz}\right)$ causes stopping of the tappet rotation (see Fig. 5b).

(a)

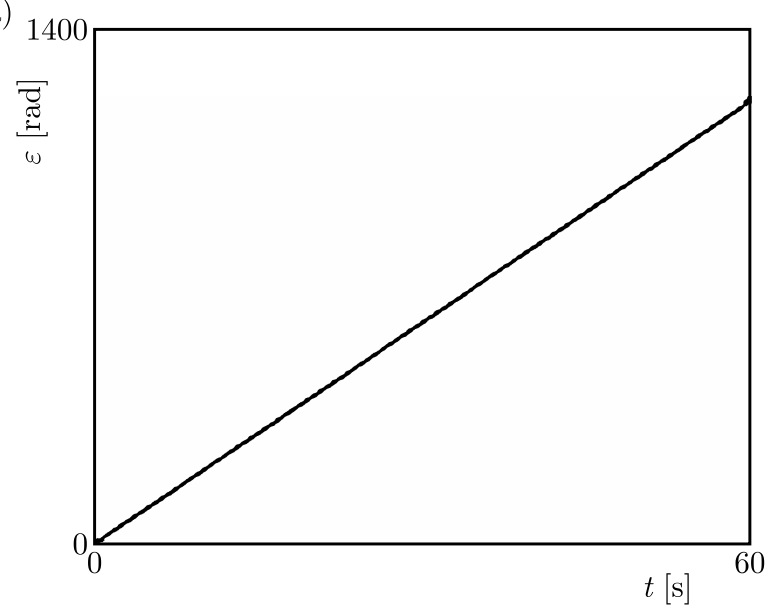

(b)

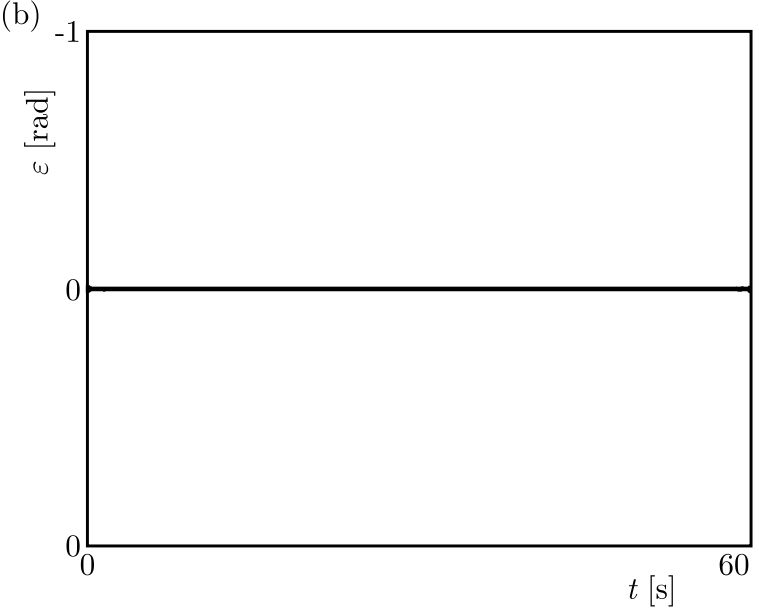

Fig. 5. Time-diagrams of the tappet rotation angle $\varepsilon$ for two different amplitudes of the camshaft longitudinal oscillations $A_{c l}: 0.0002 \mathrm{~m}(\mathrm{a}), 0.00005 \mathrm{~m}(\mathrm{~b})$. Values of remaining parameters:

$$
\mu_{c-t}=\mu_{t-p}=0.3, n_{s}=1320 \mathrm{rpm}, f_{c l}=10 \mathrm{~Hz}
$$

The results of numerical research corresponding to the case presented in Fig. 4b $\left(A_{c l}=0.0001 \mathrm{~m}, f_{c l}=10 \mathrm{~Hz}\right)$ but performed for two times larger rotational speed of the camshaft, i.e., $n_{s}=2640 \mathrm{rpm}$, are illustrated in Fig. 6. Comparison of Figs. 3b and 6 shows that such doubling of the speed $n_{s}$ does not influence significantly the rotational dynamics of the tappet. The increase in the angle $\varepsilon$ in time still has almost a linear character, and the speed of the tappet rotation is about $15 \%$ higher only.

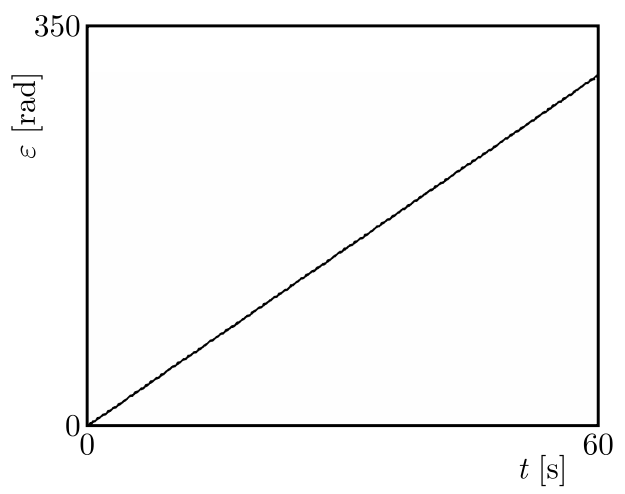

Fig. 6. Time-diagrams of the tappet rotation angle $\varepsilon$ for the camshaft rotational speed $n_{s}=2640 \mathrm{rpm}$. Values of remaining parameters: $\mu_{c-t}=\mu_{t-p}=0.3, f_{c l}=10 \mathrm{~Hz}, A_{c l}=0.0001 \mathrm{~m}$

The second group of the results have been obtained for the case (f) when the oil is present in the contact zones. Variations of mixed or hydrodynamic friction coefficients cause changes in the loading and sliding velocity of the contacting surfaces. They result in varying friction forces $F_{c-t}$ (Eq. (3.24)) occurring in contact between the cam and tappet, and $F_{t-p}$ (Eq. (3.1)) between the tappet and the push rod which have been calculated from the Reynolds equations. 
The time-diagram of the friction force $F_{c-t}$ between the cam and tappet corresponding to the graph shown in Fig. 4a $\left(n_{s}=1320 \mathrm{rpm}, f_{c l}=10 \mathrm{~Hz}\right)$ is depicted in Fig. 7a. The oil lubrication causes values of the friction forces to be a few times lower (2-4) than in the case of constant friction coefficients $\mu_{c-t}$ and $\mu_{t-p}$. Time histories of the tappet rotation angle $\varepsilon$ are shown in Fig. 7b. It is clearly visible that, similarly to the case presented in Fig. 4a, an increase in the angle $\varepsilon$ has almost a step-like character.

(a)

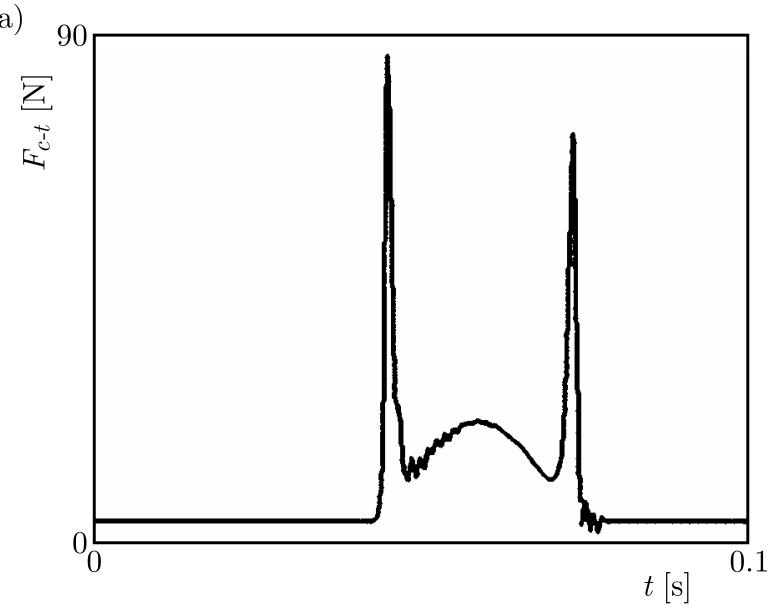

(b)

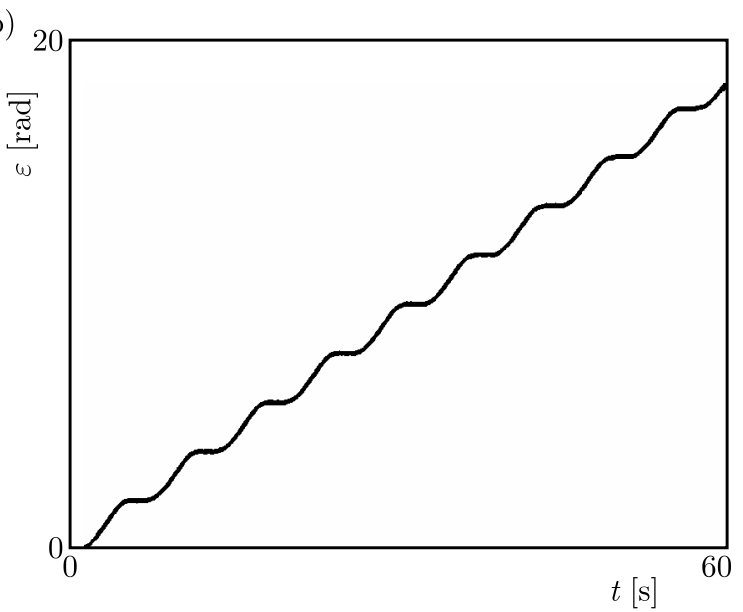

Fig. 7. Time-diagram of the force $F_{c-t}$ between the cam and the tappet (a) and the tappet rotation angle $\varepsilon$ (b) for the case of oil lubrication. Values of others parameters: $f_{c l}=1 \mathrm{~Hz}, n_{s}=1320 \mathrm{rpm}$,

$$
A_{c l}=0.0001 \mathrm{~m}
$$

In the case when the camshaft longitudinal oscillations frequency $f_{c l}$ is equal to $100 \mathrm{~Hz}$, the time history of the friction force $T_{c-t}$ between the cam and tappet is presented in Fig. 8a. Such values of the friction force are much lower, even two orders in magnitude lower than in the case of frequency $f_{c l}=1 \mathrm{~Hz}$ (see Fig. 7a). It is due to the dominant role of hydrodynamic lubrication in both contact zones, between the cam and tappet and between the tappet and pushrod, respectively. Such small values of friction forces also result in lack of tappet rotation, as it is shown in Fig. 8 b.

(a)

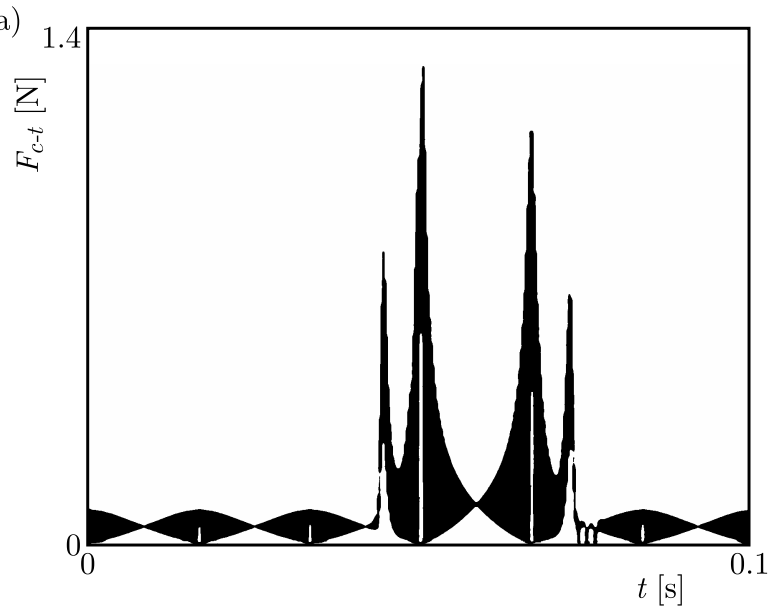

(b)

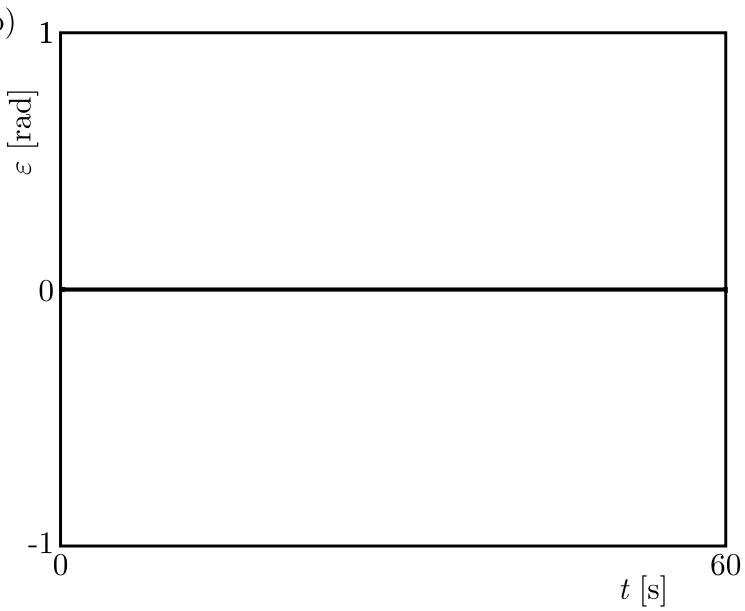

Fig. 8. Time-diagram of the force $F_{c-t}$ between the cam and the tappet (a) and the tappet rotation angle $\varepsilon$ (b) for the case with oil lubrication. Values of others parameters: $f_{c l}=100 \mathrm{~Hz}, n_{s}=1320 \mathrm{rpm}$,

$$
A_{c l}=0.0001 \mathrm{~m}
$$




\section{Summary and conclusions}

We have carried out the analysis of friction resistance between the cam and its tappet for different values of the amplitude and frequency of camshaft longitudinal oscillations and camshaft rotational speeds. Depending on these parameters or presence (or not) of oil lubrication, different time diagrams of the tappet rotation angle $\varepsilon$ are calculated and shown.

In the case of a constant value of the friction coefficient between the cam and tappet and between the tappet and pushrod, the same camshaft rotational speed and the same values of the oscillation amplitudes result with different shapes of time histories depending on the camshaft longitudinal oscillation frequency. Some results are illustrated in Figs. 4a-d. They prove that the increase in this frequency leads to a significant reduction of the average angular velocity of tappet rotation and reversal of its direction of rotation. This velocity is represented by an average slope of the time histories in Figs. $4 \mathrm{a}-\mathrm{d}-$ its value is reduced from about $50 \mathrm{rad} / \mathrm{s}$ for $f_{c l}=1 \mathrm{~Hz}$ (Fig. 4a) to about $-1 \mathrm{rad} / \mathrm{s}$ (opposite direction) for $f_{c l}=100 \mathrm{~Hz}$ (Fig. 4d). Other interesting phenomena detected during the analysis are:

- changes in the global direction of the tappet rotary motion (compare Figs. 4a,b with Figs. 4c,d),

- cyclic variations in the direction of rotation with a relatively large amplitude (Fig. 4c), which tend to zero with an increase in the frequency $f_{c l}$, then the tappet rotates slowly with an almost constant speed (Fig. 4d),

- step-like shape of the time-course of tappet rotation for small values of the frequency $f_{c l}=1 \mathrm{~Hz}$ - one can observe revolutions interrupted with stopping periods (vertical sections of the diagram in Fig. 4a) what indicates the stick-slip character of the cam-tappet dynamical contact (for am increasing frequency, the angular velocity of the tappet is stabilized - see Fig. 4b).

Analyzing the influence of camshaft rotational speed and the amplitude of longitudinal oscillations, one can draw the following conclusions:

- growing amplitude of camshaft longitudinal vibration causes a considerable increase in the tappet rotational speed (Fig. 5a), whereas its reduction to small values leads to stopping of the tappet rotation (Fig. 5b),

- even a large increase in the camshaft rotational speed does not change significantly the velocity of tappet revolutions (Fig. 6).

When the oil lubrication is applied to the contact zones between the cam and tappet and between the tappet and pushrod, rather a large reduction of the friction force and tappet rotational velocity takes place when compared to contact without any lubrication. This fact can be treated as an obvious and expected effect. The comparison with corresponding cases without lubrication (see Figs. 3 and $4 \mathrm{a}$ ) indicates that after oil application the friction force declined several times (Fig. 7a) and tappet rotations slowed at least 150 times (Fig. 7b).

However, our results demonstrate that further large reduction of friction can be obtained for a high frequency of the camshaft longitudinal vibration. Comparison of the cases presented in Figs. 7a and 8a displays that a hundredfold increase in the frequency causes almost a hundredfold decrease in the friction force. This fact can be explained by the hydrodynamic lubrication effect (squeeze effect) described in Guran et al. (1996). Hence, lack of tappet revolutions, depicted in Fig. 8b, is not surprising due to the friction force which is too weak to maintain the rotations.

We can conclude that the presented phenomena, i.e., changes in the global direction of tappet rotation, its local oscillations, the friction force dependence with the frequency and the other facts mentioned above are effects of mutual interaction of camshaft rotations with its longitudinal vibrations and possible different configuration of the phase synchrony between them. However, an accurate identification and description of the mechanism governing such phenomena requires 
more detailed bifurcation analysis in the system parameter space. This is our task for the nearest future and the results will be reported soon.

Acknowledgment

This work has been supported by Polish National Centre of Science (NCN) under Project No. DEC-2012/06/A/ST8/00356.

\section{References}

1. Baş H., BiyiklioĞlu A., Cuvaldi H., 2003, A new test apparatus for the tribological behavior of cam mechanisms, Experimental Techniques, September/October, 28-32

2. Beddoes G.N., 1992, Valve materials and design, Ironmaking and Steelmaking, 19, 290-296

3. Blair G., McCartan C.D., Hermann H., 2005, Making the Cam. Valvetrain Design Part Two. Special Investigation. Race Engine Technology, Issue 9, ISSN 1740-6803

4. Calabretta M., Cacciatore D., Carden P., 2010, Valvetrain friction - modeling, analysis and measurement of a high performance engine valvetrain system, SAE International Journal of Engines, 3, 2, 72-84, DOI: 10.4271/2010-01-1492

5. Gecim B.A., 1992, Tribological study for a low-friction cam/tappet system including tappet spin, Tribology Transactions, 35, 2, 225-234

6. Greenwood J.A., Tripp J.H., 1971, The contact of two nominally flat rough surfaces, Proceedings of the Institution of Mechanical Engineers, 185, 625-633

7. Guo J., Zhang W., Zhang X., 2015, Modeling and analysis of the transient vibration of camshaft in multi-cylinder diesel engine, Advances in Mechanical Engineering, 7, 11, 1-14

8. Guo J., Zhang W., Zou D., 2011, Investigation of dynamic characteristics of a valve train system, Mechanism and Machine Theory, 46, 1950-1969

9. Guran A., Pfeiffer F., Popp K., 1996, Dynamics with friction: modeling, analysis and experiment: (Part I), World Scientific, 23.08.1996 - 264

10. Han Y., Chan C., Wang Z., Shi F., Wang J., Wang N., Wang Q.J., 2015, Effects of shaft axial motion and misalignment on the lubrication performance of journal bearings via a fast mixed EHL computing technology, Tribology Transactions, 58, 2, 247-259

11. Hiruma M., Furuhama S., 1978, A study on valve recession caused by non-leaded gasoline measurement by means of R.I., Bulletin of JSME, 21, 147-160

12. Jelenschi L., Cofaru C., Sandu G., Aleontem M., 2011, State of the art of engine valve and tappet rotation, Bulletin of the Transilvania University of Braşov, Series I, 4, 53, 2

13. Kano M., 2006, Super low friction of DLC applied to engine cam follower lubricated with estercontaining oil, Tribology International, 39, 1682-1685

14. Kim D., LEE J., LEE J., 2004, Effect of valve train design parameters on valve rotation performance of a diesel engine, SAE Technical Paper, F2004V281

15. Kushwaha M., Rahnejat H., Jin Z.M., 2000, Valve-train dynamics: a simplified triboelastomulti-body analysis, Proceedings of the Institution of Mechanical Engineers, Part K: Journal of Multi-Body Dynamics, 214, 5-110

16. Lewis R., Dwyer-Joyce R.S., 2002, Wear of diesel engine inlet valves and seat inserts, Proceedings of the Institution of Mechanical Engineers, Part D: Journal of Automobile Engineering, 216, 205-216

17. Lindhom P., Svahn F., 2006, Study of thickness of sputtered-carbon coating for low friction valve lifters, Wear, 261, 241-250 
18. Meng F., Wang Q.J., Hua D. Liu S., 2010, A simple method to calculate contact factor used in average flow model, Journal of Tribology, 132, 2, 024505

19. Moraru L.E., 2005, Numerical predictions and measurements in the lubrication of aeronautical, engine and transmission components, Doctoral Thesis, The University of Toledo

20. Patir N., Cheng H.S., 1978, An average flow model for determine effects of three dimensional roughness on partial hydrodynamic lubrication, Journal of Lubrication Technology, 100, 1, 12-17

21. Patir N., Cheng H.S., 1979, Effect of surface roughness orientation on the central film thickness in EHD contacts, Proceedings of the 5th Leeds-Lyon Symposium on Tribology, 15-21

22. Refalo K., Bowyer S., Smith M., Hijawi M., 2010, Optimizing valve rotational speed using Taguchi techniques, SAE Technical Paper, 2010-01-1096

23. Rothbart H.A., 2004, Cam Design Handbook, McGraw Hill, New York

24. SiczeK K., 2016, Tribological Processes in the Valve Train Systems with Lightweight Valves. New Research and Modelling, 1st Ed., Elsevier

25. Soejima M., Ejima Y., Wakuri Y., Kitahara T., 1999, Improvement of lubrication for cam and follower, Tribology Transactions, 42, 4, 755-762

26. Taraza D., Henain N.A., Teodorescu M., Ceausu R., Bryzic W., 1999, Dynamics and friction of valve trains, Arc Annual Meeting, University of Michigan

27. Teodorescu M., Kushwaha M., Rahnejat H., Taraza D., 2005, Elastodynamic transient analysis of a four-cylinder valve train system with camshaft flexibility, Proceedings of the Institution of Mechanical Engineers, Part K: Journal of Multi-body Dynamics, 219, 13-25

28. Teodorescu M., Taraza D., Naeim A.H., 2003, Simplified elasto-hydrodynamic friction model of the cam-tappet contact, SAE Paper, 2003-01-0985

29. Teodorescu M., Theodossiades S., Rahnejat H., 2007, Multi-physics approach to design analysis of powertrain sub-systems, 12th IFToMM World Congress, Besançon, France

30. Willermet P.A., Pieprzak J.M., Dailey D.P., Carter R.O., Lindsay N.E., HaAck L.P. AND DEVRIES J.E., 1991, The composition of surface layers formed in a lubricated cam/tappet contact, Transaction of the ASME, Journal of Tribology, 113, 38-47

31. Wu C.W., Zheng L.Q., 1989, An average Reynolds equation for partial film lubrication with a contact factor, Journal of Tribology, 111, 188-191

32. Yang L.S., Akemi I., Hideo N., 1996 A valve train friction and lubrication analysis model and its application in a cam/tappet wear study, SAE Paper, 962030

Manuscript received April 7, 2017; accepted for print November 13, 2018 